\title{
Análise Espectral de Sinais Caóticos Gerados pelo Mapa de Bernoulli
}

\author{
Rafael Alves da Costa Murilo Bellezoni Loiola \\ Universidade Federal do ABC, UFABC, \\ 09210-170, Santo André, SP \\ E-mail: r.costa@ufabc.edu.br, murilo.loiola@ufabc.edu.br,
}

Marcio Eisencraft

Escola Politécnica da Universidade de São Paulo, USP, 05508-010, São Paulo, SP

E-mail: marcio@lcs.poli.usp.br.

\section{RESUMO}

Nesse trabalho faz-se um estudo das propriedades temporais e espectrais dos sinais caóticos gerados por uma família de mapas lineares por partes, o mapa de Bernoulli [3]. Mais especificamente, obtêm-se fórmulas analíticas para a Sequência de Autocorrelação (SAC) e para a Densidade Espectral de Potência (DEP) do sinais gerados em função do parâmetro dessa família. Abordagem para um mapa com alguma similaridade encontra-se em [4]. O desenvolvimento empregado nesse trabalho é inspirado em [2] e [5].

A família de mapas de Bernoulli é definida por

$$
s(n+1)=f_{B}(s(n))
$$

em que

$$
f_{B}(s)=\left\{\begin{array}{lc}
\frac{2}{1+\alpha} s+\frac{1-\alpha}{1+\alpha}, & -1 \leqslant s<\alpha \\
\frac{2}{1-\alpha} s-\frac{1+\alpha}{1-\alpha}, & \alpha \leqslant s \leqslant 1
\end{array},\right.
$$

sendo $\alpha \subset[-1,1]$ um parâmetro fixo. Na Figura 1(a) são mostrados gráficos de $f_{B}(s)$ para diversos valores de $\alpha$. Os expoentes de Lyapunov dos atratores desses mapas são positivos, como mostrado na Figura 1(b), dessa forma, quase todos os sinais gerados são caóticos. Na Figura 1(c) mostram-se exemplos de sinais gerados, deixando-se explícita a dependência sensível com as condições iniciais, característica dos sinais caóticos [1].
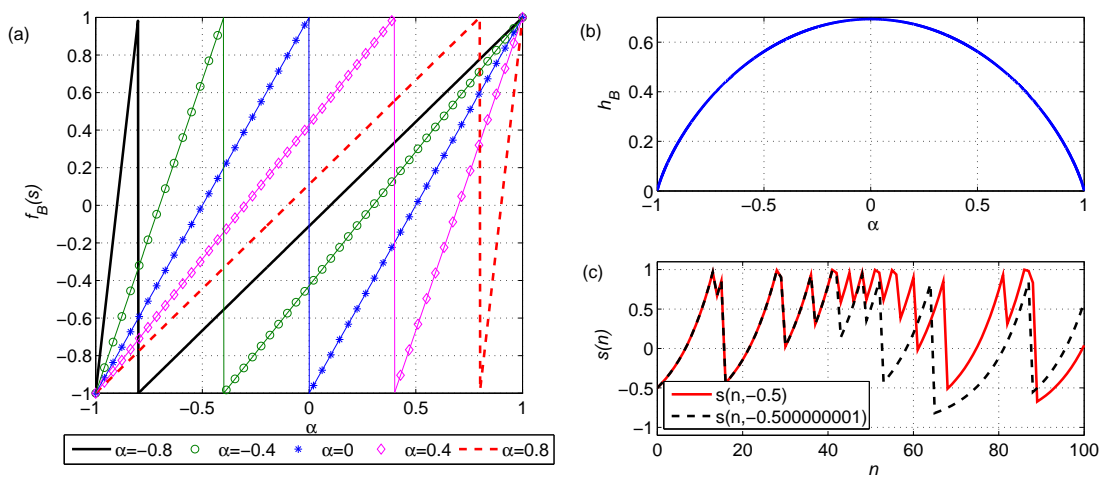

Figura 1: (a) Mapas de Bernoulli para diferentes valores de $\alpha$, (b) Expoente de Lyapunov do atrator de $f_{B}(s)$ em função de $\alpha$ e (c) trecho de órbitas de $f_{B}(s)$ com $s_{0}=-0.5$ e $s_{0}=-0.500000001$ para $\alpha=0.8$. 
A SAC $R(k)=E[x(n) x(n+k)]$, sendo $E[\cdot]$ o operador valor esperado, pode ser obtida analiticamente seguindo procedimento análogo ao descrito em [2]. Pode-se mostrar que

$$
R(k)=\frac{1}{3} \beta^{|k|},
$$

sendo $\beta=\frac{\left(1+\alpha^{2}\right)}{2}$. Para calcular a DEP $S(\omega)$ basta calcular a Transformada de Fourier de Tempo Discreto de $R(k)$, obtendo-se

$$
S(\omega)=\frac{1}{3}\left(\frac{1-\beta^{2}}{1+\beta^{2}-2 \beta \cos (\omega)}\right)
$$
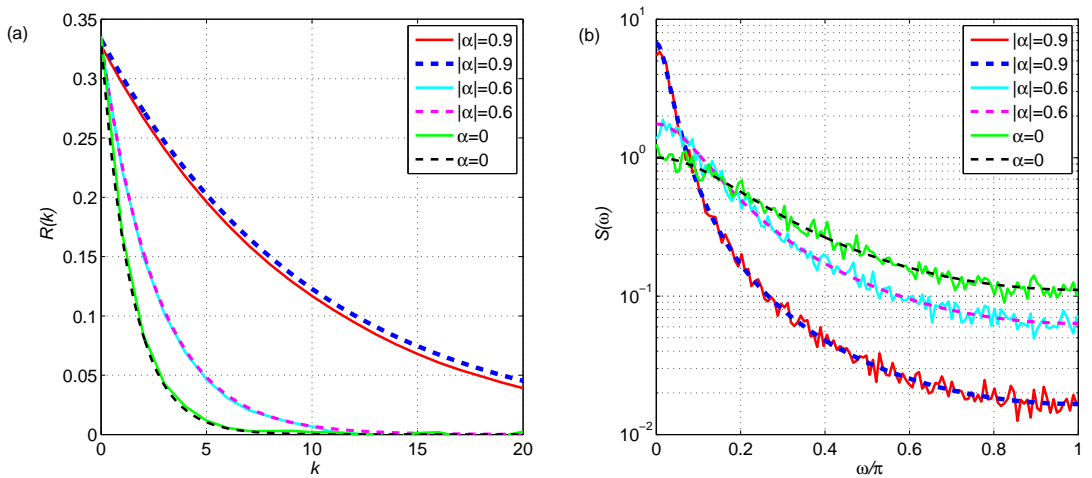

Figura 2: (a) SAC e (b) DEP para sinais do mapa de Bernoulli para alguns valores de $|\alpha|$. Em linha tracejada estão as curvas analíticas e em linha cheia as curvas obtidas via simulação numérica.

Nas Figuras 2(a) e (b) são mostrados gráficos de $R(k)$ e $S(\omega)$ para diversos valores de $|\alpha|$. Em ambos os casos são mostrados os resultados analíticos e numéricos. Nota-se que $R(k)$ decai monotonicamente, aproximando-se da forma impulsiva para $\alpha=0$, diferentemente do que ocorre com o mapa tenda inclinada estudado em [2]. Esse formato de $R(k)$ faz com que a potência dos sinais esteja concentrada nas baixas frequências para todo $|\alpha|$. Essa concentração aumenta com $|\alpha|$, podendo-se obter sinais de banda estreita para $|\alpha| \approx 1$.

Como continuação desse trabalho, pretende-se obter a banda essencial desses sinais em função de $\alpha$ e do expoente de Lyapunov. Também pretende-se estender esse estudo para mapas lineares por partes mais gerais.

Palavras-chave: Análise Espectral, Sinais Caóticos, Mapa de Bernoulli

\section{Referências}

[1] Alligood, K., Sauer, T., And Yorke, J. Chaos: An Introduction to Dynamical Systems. Textbooks in Mathematical Sciences. Springer, 1997.

[2] Eisencraft, M., Kato, D. M., And Monteiro, L. H. A. Fast communication: Spectral properties of chaotic signals generated by the skew tent map. Signal Process. 90, 1 (2010), 385-390.

[3] Lasota, A., And MacKey, M. Probabilistic properties of deterministic systems. Cambridge University Press, 1985.

[4] Lopes, A., LOPES, S., AND SouzA, R. R. On the spectral density of a class of chaotic time series. Journal of Time Series Analysis 18, 5 (1997), 465-474.

[5] Sakai, H., And Tokumaru, H. Autocorrelations of a certain chaos. Acoustics, Speech and Signal Processing, IEEE Transactions on 28, 5 (Oct. 1980), 588-590. 